\title{
Medication compliance aids: a qualitative study of users' views
}

\author{
Jan Lecouturier, Bill Cunningham, David Campbell and Richard Copeland
}

\begin{abstract}
Background

Despite the rapid rise in the use of multicompartmental compliance aids (MCAs), little is known about the role they play in self-management of medication.

Aim

To explore the perceived benefits of MCAs for people using them to manage their own or a relative's medication.

\section{Design of study}

Qualitative study using in-depth interviews.

\section{Setting}

West Northumberland.

\section{Method}

Recruitment was via posters and leaflets in general practices and community pharmacies. In-depth interviews were conducted using a topic guide.

Results

Nineteen people were interviewed. Three overarching themes emerged in relation to medicine taking: disruption, organisation, and adherence, which impacted on control. The medication regime had caused disruption to their lives and this had led to the purchase of an MCA. The MCA enabled them to organise their medication, which they believed had improved the efficiency of medicine taking and saved time. Although the MCA did not prompt them to take their medication, they could see whether they had actually taken it or not, which alleviated their anxiety. To meet their individual needs and lifestyles, some had developed broader systems of medication

management, incorporating the MCA. For a small cost - the initial outlay for the MCA and time spent loading it - they gained control over the management of their medication and their condition.

\section{Conclusion}

This group found the use of an MCA to be beneficial, but advice and support regarding how best to manage their medication and on the most appropriate design to suit their needs would be helpful.
\end{abstract}

\section{Keywords}

medication adherence; medication systems; qualitative research.

\section{INTRODUCTION}

Multicompartmental compliance aids (MCAs) are reusable plastic containers for taking medicines, that are divided into days/time of day. A community pharmacist, the person taking the medicine, or a carer may dispense or place medicines into the MCA. A monitored dosage system (MDS) has an individual compartment for medicines to be taken at a set time each day, is disposable, and is dispensed by pharmacists as a blister pack or roll of sachets. Both require some manual dexterity and, although there are some MCAs on the market with alarms and/or text reminders, most do not prompt the person to take the medication. They are labour intensive as they require individual doses to be loaded into the appropriate compartment. This introduces the risk of errors no matter who carries out this task. For reusable MCAs there is the added risk of medicines being dropped from compartments and replaced into the wrong one, thus compounding the risk of unintentional non-adherence. There are doubts about the benefits of these devices, as there is little evidence of effectiveness,,$^{1-3}$ and there are concerns regarding the stability of medicines in MCAs/MDSs once moved from their original packs. ${ }^{4}$

Acute trusts in England, often in conjunction with primary care trusts, have sought to develop policies to help determine which patients may benefit from the use of MCAs. ${ }^{5}$ The contractual framework for

J Lecouturier, BSc, MSc, senior research associate, Institute of Health and Society, Newcastle University, Newcastle. B Cunningham, FRCP, FRCGP, GP principal, Corbridge Medical Group, Corbridge. D Campbell, BSc, DipHSM, MBA, MRPharmS, chief pharmacist/clinical director for medicines management; $\mathbf{R}$ Copeland, MPhil, MRPharmS, lead clinical pharmacist: clinical governance, Pharmacy Department, Northumbria Healthcare NHS Trust, North Shields,.

Address for correspondence

Ms Jan Lecouturier, Institute of Health and Society, Newcastle University, Newcastle, NE2 4AX.

E-mail: jan.lecouturier@ncl.ac.uk

Submitted: 21 May 2010; Editor's response: 15 June 2010; final acceptance: 27 July 2010.

(c)British Journal of General Practice 2011; 61: 93-100.

DOI: 10.3399/bjgp11X556191 


\section{How this fits in}

Multicompartmental medication aids are increasingly used as a way of improving adherence/compliance and to deal with increasingly complex regimes. However, this is happening in an uncoordinated way without clear evidence of benefit. There is a need for a greater understanding of the role these devices play in the management of medication for those living in the community. study leaflets to appropriate patients. Those who were interested completed and returned the reply slip to the researcher. The patient was contacted to answer any questions and, if appropriate, arrange an appointment to obtain consent and conduct the interview. Most interviews were conducted at the patient's home.

\section{Analysis}

Data were analysed using a framework approach (Box 1). ${ }^{5}$

community pharmacists in England, introduced in 2005, included a requirement for pharmacists to assist patients in enabling compliance, and to include the provision of compliance aids, if deemed to be a 'reasonable adjustment'.

This exploratory study seeks the views of those who use, or have previously used, an MCA/MDS, on the perceived benefits in helping them to manage their own or a relative's medication.

\section{METHOD \\ Design}

This was a qualitative study using in-depth interviews. A topic guide was used but interviewees were encouraged to talk freely and raise any other issues related to medicines management. The interviews were designed to explore the costs and benefits of using an MCA. Descriptive data were obtained, for example, the pathway to obtaining an aid and its use in daily medication management. Views on the benefits and drawbacks as well as barriers and facilitators to the use of an MCA were explored. Interviews were recorded and transcribed verbatim. Transcripts were checked against the original sound file for errors.

\section{Setting}

Posters with study leaflets were displayed in community pharmacies and general practices in West Northumberland, and district nurses distributed

\section{Box 1. Description of the process of analysis.}

- Familiarisation: listen to sound files and read transcripts from 10 interviews to look for recurrent themes emerging from the data

- Develop a thematic framework: emergent themes from the data along with issues and questions related to the original aims and objectives of the studies are brought together into a framework

- Test and refine the framework: the framework is applied to a 'new' batch of interview transcripts and amended accordingly

- Coding: transcripts are imported into NVivo7 and each transcript is coded using the final revised framework

- Mapping and interpretation: using the model function in NVivo7, themes are mapped to look for associations and define concepts

\section{RESULTS}

Nineteen people, aged from 20 s to late 80 s, were interviewed (Table 1). In four interviews a second person participated: two managed the interviewees' medication, the third shared the use of the MCA, and the fourth no longer used one. Due to the nature of recruitment, interviewees were not asked about their medical history, although some volunteered this information. Over half took 6-18 tablets per day and 20 years.

Three overarching themes emerged in relation to medicine taking and MCA use: disruption, organisation, and adherence. In the following sections, the pathway to obtaining an aid is described; the three themes are considered in more depth and their impact on control examined; and finally some of the practical issues raised are discussed.

\section{Pathway to obtaining an aid}

Most patients obtained an MCA because they found their treatment regimen difficult to manage effectively, due primarily to the complexity and/or amount of medication, but in some cases to memory problems. This was as much a problem for those managing the medication of a family member:

'[It's] less than 10 years since I started having to use pills to sort of have control of the diabetes and when I got the ... cancer, the operation, and everything went absolutely haywire and so there was more pills and more pills so that's when I started using [MCA] to try and control it ...' (patient, interview 12)

'... because you can imagine, 17 [types of medication], some were twice a day and some were three times a day and some were just night, so it was quite difficult, so this is where this (carer, interview 17)

Mostly the interviewee or a family member had (Table 1). Aids had been used for between 6 months [MCA] came in. There was no mistake then.' 
purchased the MCA. In three cases a district nurse, a nurse specialist, and a home help had suggested it may be beneficial. Apart from two who initiated discussions with a community pharmacist before purchasing an MCA, no one mentioned to their GP or pharmacist that they were having difficulties. Conversely, none could recall any of the health professionals asking whether they were able to adequately manage their medication. One person described leaving hospital supplied with two small plastic cups - used to dispense medicine on the wards - to help him cope.

\section{Emergent themes}

Disruption - bringing order to chaos. The treatment regimen had caused disruption to the lives of interviewees and families. Some were anxious and others overwhelmed by the task of managing their medication. One person described being 'in a muddle' when prescribed warfarin, and anxious about how she would cope with this and her existing medication:

'But I really was worried about these, because I thought well I can't remember what I was taking, you know, if I have to go into a box every time and get them out, goodness knows how I would've managed because taking three or four different ones each in the morning, afternoon, you know?' (patient, interview 4)

Some could not remember how many of each drug to take at the various time points and had to check every time. Unless they were systematic about the task, those with memory problems could forget very quickly which ones they had taken:

'It was stressful and [I] just had a bag full of boxes and have to look at the front three times a day.' (patient, interview 16)

'So we're talking three, four, five, six ... If I was taking them one at a time ... I would get halfway through the various packets and think "have I taken that one or not?", my memory gets as confused as that. Probably also because I'm trying to do half a dozen other things at the same time, and make tea, talk to kids, sort dogs out and stuff like that, and so I often lose track of what I'm doing.' (patient, interview 14)

This disruption extended to other family members. One person described the level of support he required daily to ensure he complied with his medication:

'... I had to get up with [partner], take my
Table 1. Participant details.

Characteristic

\begin{tabular}{lc}
\hline Sex, $n$ & \\
Male & 8 \\
Female & 11 \\
\hline Work status, $n$ & \\
$\quad$ Retired & 10 \\
Unable to work & 4 \\
In full-time employment & 3 \\
Looking after family and home & 2 \\
\hline Medication, median (range) & \\
Medications prescribed per person (tablet) & $5(2-9)$ \\
Tablets taken daily & $6(4-18)$ \\
\hline Compliance aid & \\
MCA & 17 \\
MDS (from pharmacist) & 2 \\
\hline
\end{tabular}

$M C A=$ multicompartmental compliance aids .

$M D S=$ monitored dosage system .

morning ones, and then she was going through every box trying to read it, which one was for the morning ... dinnertime, tea, supper ... she put them up for like tea and supper [laughs] ... my [relative] was having to come over from work on his dinner break and make sure l'd had my tablets, and then he was coming over when he finished work at tea time because [partner] sometimes doesn't get back until after 6.' (patient, interview 16)

Administering medicine directly from the original packaging three or four times a day was timeconsuming as well as difficult physically. The MCA reduced the number of occasions patients dispensed from the original packaging and they believed that this, in turn had improved the efficiency of their medicine taking:

'I mean some of the branded things come in very, very tight screw-top things. And I have to get bottle openers to open them or wait for somebody to come.' (patient, interview 6)

'When I started using that box it revolutionised the pill taking in the morning because [previously] it took me so long to get each packet out ... remember what day it was, is it my methotrexate day is it my folic acid day? No, because I only take those one day a week. Um, and just fiddly and pills flying and everything.' (patient, interview 7)

People's work and social lives were disrupted. The original packaging was impractical to take outside of the home and it was 'a matter of putting them in a little pot, or whatever'. One interviewee said her 
husband had previously taken his medication to work in his lunch box when it often became 'mixed in with his sandwiches'. With some MCAs, they could remove one segment which would easily fit into their pocket or handbag:

'I was suddenly bombarded with quite a number of tablets to take ... I had a very busy job which involved starting early and finishing very late, and it really wasn't practical to take around packets of pills and all the rest of it, and so I needed to have something that I could carry around medication quickly and simply.' (patient, interview 18)

Similarly, people used their MCA to manage their medication when they went away on holiday so they did not have to transport boxes and bottles of medicines:

'So when I'm going on holiday for 2 weeks, I put the 2 weeks' worth of tablets in the box, and so I always know l've got my tablets with me and I keep it in my handbag for getting through airports and stuff.' (patient, interview 14)

Use of the MCA reduced disruption and enabled people to lead more normal lives in other ways. It minimised the visibility of the medication, reduced the stigma of taking medication and of being 'ill', and reduced their dependence on others:

'I feel much less of an invalid because the taking of the pills, the whole process of it, is much, can be much more discreet and much speedier each day.' (patient, interview 7)

'It's not anybody else's business in the house what's going on with my tablets ... this is ... something that I can control myself, and it's not them looking out for me because l'm brain damaged, which is a stigma obviously l've not been comfortable with. And at least with the box I can kick myself for not having taken them, but nobody else particularly needs to know, it's not relevant to anybody else.' (patient, interview 14)

Organisation - empowering patients and informal carers. The MCA helped to organise medicine taking in a number of ways. As mentioned previously, it streamlined the daily administration of medicine. It was also invaluable in enabling people to manage varying dosages, medicines not required every day, or temporary breaks in their regimen:

'When I had to take $150 \mathrm{mg}$ on one day and
175 mg every other day ... I just couldn't begin to do that without a box of tricks to sort me out. And then of course it's a different day over the week, because it's every other day and the week's an odd number of days.' (patient, interview 7)

'[Partner] takes another tablet in the morning for thinning the blood, and it's quite a special tablet ... it's for heart trouble. But ... that has to be stopped, prior to going in for surgery ... so again, I then just leave that one off, for the week prior to him going in and the week after he comes out, otherwise it would cause bleeding in the stomach.' (carer, interview 17)

Some also used the aid to facilitate adherence to vitamins. Others preferred to keep vitamins and medicines that were not taken daily separate. There were mixed feelings about incorporating short-term medications such as antibiotics in the MCA: some kept these next to their MCA so they would remember to take them.

People were able to plan ahead with the MCA. One person could look ahead and adjust her medication and tailor dosages according to an impending situation:

'And I can change my dosage in the box so easily ... So for example Prozac ${ }^{\circledR}$ tablets ... I'm either on two a day or three a day, and so I can plan my week ahead, know what are going to be the stressful points, and make sure for that morning, that I put three in. You know, if it's going to be a fairly normal week I will have kept it back to two. So for example weekends I know that with possibly both of my kids around, and a husband flitting in and out, things will be far more stressful and far more complicated.' (patient, interview 14)

Another major benefit of advance preparation was knowing when to renew their prescription. Previously, it was difficult to tell if they needed to restock one or more of their tablets:

'Well I tend to get a 3-month supply at a time, which means one of my cupboards in the kitchen is a bit like a pharmacy itself, you know, it's got piles of stuff in. And because I take so many different ones I think there are about 13 different tablets and they never all run out at the same time, you know ... I do find it helps me keep a better track on when one or more of the tablets are ... needing renewing.' (patient, interview 19) 
'If you're putting them up for the next week ... and you only get as far as Thursday and you haven't got any left it makes you think "oh I need to put another prescription in" ... Whereas if you're just taking them out of the packet, especially with them being in normal blister packs you might always think "oh there'll be another pack there" and there's not.' (patient, interview 8)

Adherence - confidence and reassurance. This was a well-motivated group of people who recognised the importance of adhering to their medication regimen; most had sought ways to improve adherence of their own accord. A major worry with conventional packaging was they could not always tell whether or not they had taken their medicine. One concern was overdose when they had forgotten they had taken a drug and then taken it again:

'I'm far more likely to overdose or to completely forget tablets without something like [MCA] to organise me, because I won't know whether l've taken the damn thing or not. The number of times, by the time l've actually swallowed the tablet and think "Yes, I do remember doing that this morning. Oh well, too late now" and so yes, it's my reassurance.' (patient, interview 14)

'You'll know if you've missed a tablet because it's still there. And you can't take too many, which is a very big danger with older people ... because the box has only got the correct number in for you to take. So from a medical safety point of view it's brilliant.' (patient, interview 1)

According to the interviewees, none of the patients were taking medication that would be life-threatening if they were to miss a dose, but there was concern about their condition(s) not being managed or controlled effectively. Knowing whether they had taken their medication allowed them to take the necessary steps should they have missed some:

'The other one is an HRT [hormone replacement therapy] tablet, which again causes problems if I forget to take it because you suddenly get intermittent bleeding and stuff, and you start worrying about that. And then the other ones are thyroxine and so you're keeping a regular but steady dose of that is fairly important. So this bails me out on many occasions.' (patient, interview 14)

Some had devised routines or systems to complement MCA use. Many linked medicine taking to events during the day such as their first cup of tea or brushing their teeth at night. After his morning medication, one man put the remainder in a different small container to keep in his pocket as he went about his day.

\section{Control}

There was a sense that previously the process of managing their medicine had taken over the lives of many interviewees. The combination of difficulties organising and adhering to their medication caused disruption and led to a sense of loss of control for many (Figure 1). However, the introduction of an MCA had lessened this to a major degree and allowed people to put it out of their minds and get on with their lives (Figure 2). Gaining control over their medicine taking - reducing disruption, improving adherence, and streamlining the process - allowed people to lead more normal lives and lessened their dependence on others:

'I don't really think about what l've got to take, it's put up and there, and l've got a morning supply and I've got an evening supply and I don't have to think about it.' (patient interview 18)

'... of a morning you just tip them out into your hand ... and move on. Um which is just fantastic because it's not slowing up your life at all.' (patient interview 7)

\section{MCA/MDS use}

All but two of those using an MCA loaded it themselves. They tended to do this once a week or month depending upon the MCA(s) they used. Some

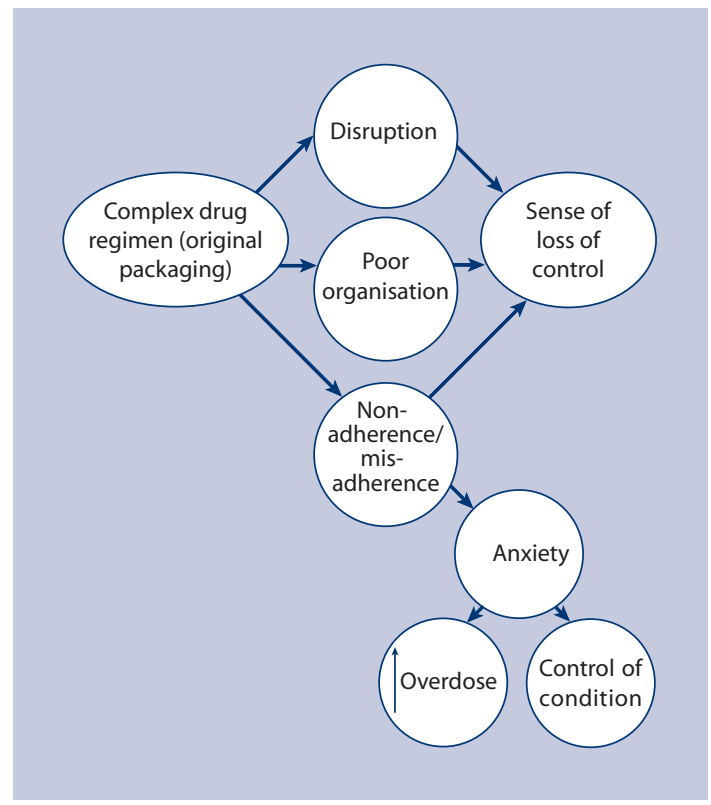

Figure 1. Impact of complex drug regimen. 


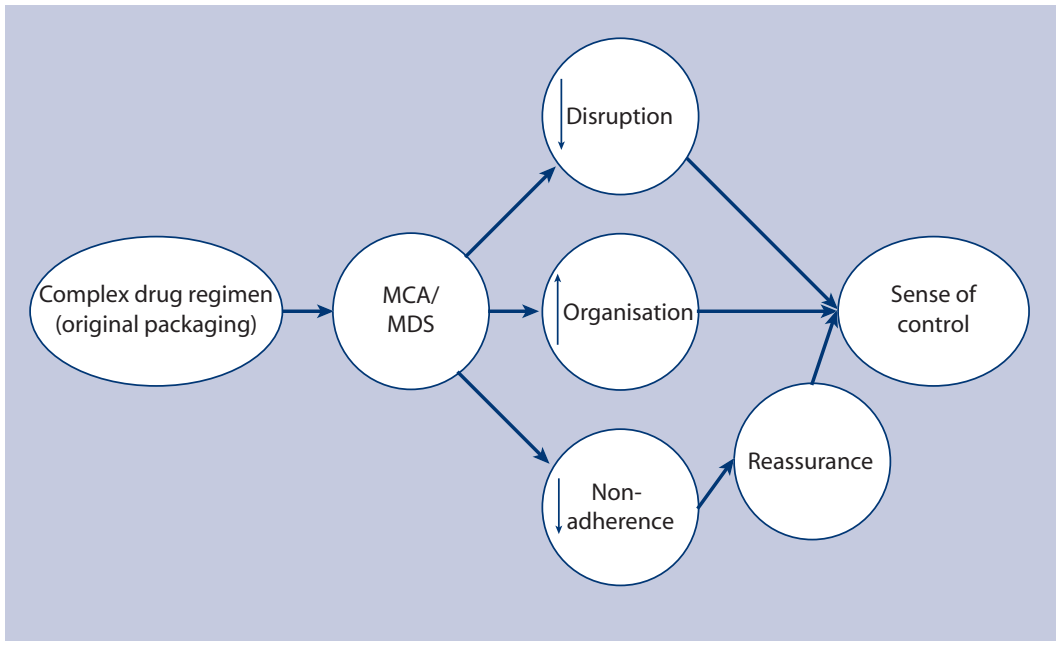

Figure 2. Impact of introduction of an MCA.

sat down and methodically worked through loading the device when they had some peace and quiet:

'If you sit down with all ... the cards there, and I do it systematically, I put the first one into every box, then the second one into every box and the third one into every box and when they're all finished I check in every box to see that the right number are in there. So it's not a life-saver, but it's just another thing off your mind.' (patient interview 9)

A few were not as systematic, citing different reasons. One person said with three young children it was difficult to establish a set time for loading the MCA and that she tended to do so when she could find ' 10 minutes of clear concentrated time'. Another merely disliked carrying out the task and found it 'a bind'. Finally, one person who had suffered a head injury found sorting the medication into the MCA very tiring and often postponed the task:

Interviewer: 'Do you prepare them on a Monday or a Friday evening, how do you ...?'

Patient: 'That's a very good question, it's one of the jobs I hate doing and I put off as long as I can, I get in a right muddle because of it. Um ... it would be lovely to think I did it on a special day each week, but I don't [laughs]).' (patient interview 6)

When asked if they ever made errors when loading the aid, there were complaints about dispensing pills from blister packs as they can fly out onto the floor or even into the wrong compartment. The majority view was that if you approach the process methodically it is actually less prone to error than taking medication from the original packaging several times a day:
'And if you set it all up at the same time, for 28 days, you're much, much less likely to make mistakes because you're looking into the boxes and you can see exactly what's in there.' (patient, interview 1)

'Instead of sitting down say every day ... you can sit once a week and have a look and take your time and make sure it's right, and once they're in you're happy enough with it, you know?' (carer, interview 17)

All used, or had used, a variation on the basic reusable MCA. No one had an aid with an inbuilt alarm or timer. People had tried different designs and found advantages and disadvantages with each. There were comments about compartments being too small, but most appreciated that if they were made larger this would make the device too big and cumbersome should they need to take it around with them. The other comments were related to lids and hinges wearing out, often because the devices were actually quite old. None were considered perfect but were thought to be the best available, and a number of people had a second device they used in different circumstances, for example if they were travelling.

Two people received their medicine weekly from the pharmacist in an MDS; an A4 card folder with four large blisters for each day of the week and medicine details recorded on the back. One had previously had his MCA loaded by the pharmacist but was asked to try out the MDS:

'The chemist just updated everything, and she said that you know, it's security for them. And I take it back ... the first week I didn't take it back and she said "no", she would like me to take it back to see I was using it properly.' (patient, interview 2)

He found it reassuring to have the details of the medicine clearly recorded on the folder. However, if the patient had to take medicine while he was out, the size was impractical and he wrapped the pills in cling film. He had now purchased a small MCA that could be carried discreetly.

The other person was less satisfied and had been transferring the medication into an MCA. Again the size was mentioned but there were also difficulties getting the medication out of the blisters:

I've had problems getting them out of the card things, actually ... the back it's really thick ... I've had like scratches to my finger in actually ... you know and then there's pills sticking underneath, 
and oh, it's just ... I thought it was really bad.' (patient, interview 16)

Also, medicines from the same blister pack had to be taken at different times, for example one at $4 \mathrm{pm}$ and the others at $6 \mathrm{pm}$, or some before and some after eating. With an MCA they could take out what they needed and close the compartment afterwards whereas with the MDS 'they're sort of lying in an open blister pack and we've got kids'.

\section{DISCUSSION}

\section{Summary of main findings}

For many interviewees, their lives were previously dominated by the medication and by concerns regarding adherence. Despite their failings, MCAs reduced the daily disruption of medicine taking by enabling interviewees to organise the process to suit their own situation and lessened concern around adherence. Gaining control over medicine taking restored control of their lives, and the management of the medical condition(s). These findings were the same for those managing their own or a family member's medication.

MDSs and MCAs are typically associated with older people, but younger people in full-time work or running a home with young children had encountered the same problems managing their medication and found these aids invaluable in the same respect as the other interviewees. Comments about MDSs raise questions over the design and suitability for those not confined to their homes and who have young children.

A number of people used an MCA when away from home but none raised concerns regarding the stability of medicines or the safety of transporting them without labels. One couple shared the same MCA but as they knew the colour and size of their own tablets did not consider there was any risk. However, as others mentioned, when pharmacies change suppliers some medicines may change in size, shape, and colour.

It was perhaps surprising that these patients' problems were not identified by health professionals or reported by the patients, given that a medication review should have been undertaken for all those taking four or more medicines, as part of the Quality and Outcomes Framework. The account given by one interviewee about being discharged from hospital with multiple medications and two of the cups used on the wards to dispense medicines which the patient and their partner found wholly inadequate - illustrates the need for advice and support for patients in the secondary care setting as well.

It is difficult to say, from the findings of this study with such a heterogeneous sample of people, if there is a 'type' of patient who is best suited to a device such as an MCA or MDS. Some obvious groups would be those with memory problems, those with busy lives - inside and outside of the home - who need to take medication at different points during the day, and those with more complex medication regimes, for example where the doses vary from day to day. What the group had in common as a whole was that they often forgot to take their medication or could not remember whether or not they had taken it.

The focus of this study was not specifically to explore views on different designs of MCA. However, some interviewees did comment briefly on their current and previous devices. As stated earlier, there were issues around the size of the compartment, and the one person interviewed who no longer used an MCA gave the reason that one of her pills would not fit into the device. Those who commented on this did not want to change the overall size of the MCA by making the compartments larger. Other comments were about lids that opened, or compartments that slid out, involuntarily, in most cases due to the fact that the device was old. Interestingly, considering that most still occasionally forgot to take their medication, no one mentioned the potential benefit of a device with a built-in alarm. Again it is difficult to point to one particular design as the ideal, as different people had different needs, or a preference for a certain type.

\section{Strengths and limitations of the study}

No other study has explored qualitatively the perceptions of people living in the community in relation to these widely used aids. This study reports the views of younger people, a group that has been neglected in previous research. Although not widely supplied at the time of this study, it obtained the views of people on one particular MDS.

It is not possible to report the perspectives of exusers on the barriers to using an MCA, although data were collected from the spouse of one interviewee whose medication was too large for the compartments. Finally, this was a self-selected sample which may have been more supportive of MCA use, although few thought the design was perfect.

\section{Comparison with existing literature}

The findings of this study are mirrored in a Canadian survey of people aged $\geq 45$ years, which reported that people used an MCA for convenience, due to difficulties adhering to their medication, and because of a complicated regimen. ${ }^{6}$ Despite missing doses, responders believed both MCA and blister packs 
facilitated adherence. A UK study within primary care reported a lack of assessment before the decision was made to use a compliance aid; ${ }^{7}$ the majority of patients had no input into the decision, or even which device was used. However, most patients who received an MCA preferred it to ordinary bottles, although this was not explored further. Participants in this study were emphatic about inherent problems with the original packaging, especially in relation to risk and in physically accessing the medicines.

\section{Implications for future research and clinical practice}

MCAs are clearly of benefit to a particular group of people who are motivated to adhere to their medication, but have struggled to manage it. A recent discussion highlights the increasing burden of treatment in patients with chronic disease and advocates a greater patient role in prioritising which aspects of treatment should be managed, and in what order. ${ }^{8}$ Effective interventions need to be found to reduce this treatment burden. ${ }^{9}$ Compliance aids may assist patients in managing the burden but it is unclear whether other interventions, such as medication reminder cards, would have a similar value. Medication review with the GP or community pharmacist would enable discussion of how best to manage their medicines, including safety issues and the most appropriate designs of aid to suit their needs.

This exploratory work has shown perceived benefits associated with the use of MCAs but raises questions about their use and design. There are some key areas that warrant future research: first, to investigate in a large-scale survey whether the experiences of those who took part in this study reflect those of the wider population, and to assess the burden of polypharmacy and its impact on perceptions of MCA use; secondly, to conduct exploratory work to determine which patients are most likely to benefit from MCA use and how healthcare professionals can recognise those who may experience problems managing their medication; thirdly, to obtain the views of healthcare professionals on the issues raised around MCA use and look at ways of providing support to people such as those who took part in the study; and finally, to examine, in a trial, the relative effectiveness and perceptions ascribed to MCA use against other strategies for medication management.

\section{Funding body}

This study was funded by the Royal College of General Practitioners (SFB/2004/19). The funding body had no involvement in the research process or in the writing of this article.

\section{Ethical approval}

Ethical approval was given by Northumberland Local Research Ethics Committee (REC Ref no: 05/Q0902/19) on 11 April 2005.

\section{Competing interests}

The authors have stated that there are none.

\section{Acknowledgements}

We thank all of the patients and their family members for taking part in this study, and the community pharmacists and district nurses for helping us to conduct this research.

\section{Discuss this article}

Contribute and read comments about this article on the Discussion Forum: http://www.rcgp.org.uk/bjgp-discuss

\section{REFERENCES}

1. Rivers P. Compliance aids — do they work? Drugs Ageing 1992; 2(2): 103-111.

2. McGraw C, Drennan V. District nurses and medication compliance devices. Pharm J 2000; 264: 368.

3. Moisan J, Gaudet M, Gregoire J, Bouchard R. Non-compliance with drug treatment and reading difficulties with regard to prescription labelling among seniors. Gerontology 2002; 48(1): 44-51.

4. Church C, Smith J. How stable are medicines moved from original packs into compliance aids? Pharm J 2006; 276: 75-81.

5. Ritchie J, Spenser L. Qualitative analysis for applied social policy research. In: Burgess BA (ed). Analysing qualitative data. London: Routledge, 1994: 173-194.

6. Gould ON, Todd L, Irvine-Meek J. Adherence devices in a community sample: How are pillboxes used? Can Pharm J 2009; 142(1): 28-35.

7. Nunney JM, Raynor DKT. How are multi-compartment compliance aids used in primary care? Pharm J 2001; 267: 784-789.

8. May C, Montori CM, Mair FS. We need minimally disruptive medicine. BMJ 2009; 339: b2803.

9. George J, Elliot RA, Stewart DC. A systematic review of interventions to improve medication taking in elderly patients prescribed multiple medications. Drugs Aging 2008; 25(4): 307-324. 